\title{
Development of Brief Image Quality Evaluation Criteria for Digital OrthoPantomography (OPG) Images in Dental Radiography
}

\author{
A.M.C. Kumarihami ${ }^{1}$, S.D.L. Heshani ${ }^{1}$, P. Sathyathas ${ }^{1}$ and R.P. Illeperuma ${ }^{2}$ \\ 1. Department of Radiography \& Radiotherapy, Faculty of Allied Health Sciences, General Sir John Kotelawala Defence University, \\ Rathmalana 10390, Sri Lanka
}

2. Department of Medical Laboratory Science, Faculty of Allied Health Sciences, University of Peradeniya, Peradeniya 20000, Sri

Lanka

\begin{abstract}
An OPG (orthopantmography) is an extra-oral radiographic imaging method which provides a panoramic or wide view of both jaws and teeth on a single image. Digital OPG images provide high contrast with more details of the dentitions. The research main objective was to produce sophisticated and effective criteria that can be used by any radiographer with sound knowledge to identify common errors of digital OPG images and to increase the concern of high frequency of errors to minimize them to give an optimum image quality. The study was designed as retrospective cross sectional study. Hundred digital OPG images are evaluated by three qualified radiographers who had dental radiography experience and four student radiographers. Paired $t$-test was used to see the difference between the responses of radiographers and student radiographers. Kruskal-Wallis Test was used to see difference between each evaluator. Possible errors of OPG were divided into four main categories (identification, artifact, anatomical coverage and patient positioning). Each main category consists of sub-categories. Values of subcategories were given according to their importance to get the total of $100 \%$ for each main category. The results showed that there is no significant difference between radiographers and student radiographers' responses and also between each evaluator. Hence it shows that the criteria were an easy understandable and user-friendly tool. And results showed the frequent error category was loss of anatomical coverage and frequent error was absence of positioning the tongue against the palate.
\end{abstract}

Key words: OPG, radiography, dental radiography, evaluation criteria.

\section{Introduction}

Dental radiography is the art of recording images of a patient's oral structures on film by using X-rays [1]. There are two main methods in imaging the oral structures according to the place of the films. In intra oral radiography film is placed in inside of the mouth and in extra oral radiography film is placed outside of the mouth. Dental panoramic radiography is one of the methods of extra oral radiography.

Dental panoramic radiography is a unique extra oral film technique that allows the dentist to view the

Corresponding author: R. P. Illeperuma, BDS, MSD, $\mathrm{Ph} . \mathrm{D}$., senior lecturer, research fields: oral cancer, mechanism of carcinogenesis. entire dentition and related structures [2]. Quality assurance of dental panoramic radiographs is very important as properly planned quality control tests and quality management programs contribute to producing a good quality image. Good quality image is the basic means to proper diagnosis.

Dental panoramic radiography imaging is mostly used for orthodontic assessments. Therefore image quality should not be minimized to avoid misinterpretation. In panoramic imaging both principal of tomography and principal of scanning are used [3]. Hence correct positioning of the dental arch inside the focal trough is important to obtain images with high diagnostical value. Images can be obtained as plain film radiographs and digital radiographs. 
There are different ways and methods to assess the quality of an OPG (orthopantomography) film. Around the world in different clinical setups and with different equipment facilities, many researchers have assessed the quality of the OPG films and many of them have observed the occurrence of large number of errors throughout the process of the production of panoramic radiographs [4]. According to the depth of our knowledge there only few researches have done on quality evaluation of panoramic images in Sri Lanka [2].

This study main purpose was to develop brief image quality evaluation criteria for OPG in dental radiography for radiographers. Each factor in quality evaluation criteria contributes in different proportion to the overall image quality. The study focused to zoom out common errors related to OPG images and the results can be used to minimize those possible errors. Minimizing image repetition directly affects in reducing patient dose [5]. Also time reduction of both patients' and the hospital cannot be neglected as time is the best source of money.

\subsection{Objectives of the Study}

The objectives are to develop brief image quality evaluation criteria for digital OPG in dental radiography for radiographers, develop a brief quality assessment criteria list to assess the quality of OPG films in accordance with the established criteria, identify the most frequent errors occurring during the panoramic radiography procedure and evaluate quality assessment variations between undergraduate student radiographers and experienced radiographers.

\section{Material and Method Research Design}

A retrospective cross sectional study was used to develop brief image evaluation criteria for OPG in dental radiography. The approval was obtained from administrative boards of respective hospitals and ethical review committee of General Sir John Kotelawela Defence University approved the study.
The study represented all the digital OPG images that have been taken from January to December in 2015 at one government hospital and one private hospital in Colombo, Sri Lanka.

\subsection{Sampling and Sample Size}

Systematic sampling method was used to select digital OPG images. The study used the largest possible sample size (100 OPG images) referring from previous similar studies. According to the population ratio 75 of images were selected from the government hospital and 25 of images selected from the private hospital. Patients without anterior teeth in the images were excluded as their dental arch cannot be positioned in focal trough.

\subsection{Method of Image Evaluation}

Images which were selected for the pre-test or pilot study were limited to six. In this study three BSc degree radiographers with more than two years' experience in dental radiography and final year undergraduate students of General Sir John Kotelawela Defence University (two male students and two female students) were selected for image evaluation. Each image (100) was given to every evaluator who was asked to observe each image according to the data collection tool (Table 1). Adequate time was given to access each image. Evaluators were asked to put tick to "NO" if the error exists in the image according to their own observation. Radiographers from Peradeniya dental hospital, Maharagama dental institute, Sri Lanka and Ward place dental institute, Sri Lanka and Student Radiographers from General Sir John Kotelawala Defence University evaluated the OPG images.

\subsection{Equipment and Material}

The OPG equipment was Owandy I-Max touch 3D machine which can perform digital panoramic imaging and 3D cone beam CT. The machine already contained software which facilitates all the functions of the 

(OPG) Images in Dental Radiography

procedures. Features of the equipment are $220-240 \mathrm{~V}$ and $50 / 60 \mathrm{~Hz}$ and max exposure time is 14 seconds.

\subsection{Statistical Analysis}

Minitab version 14 was used to analyze data with 0.05 significant levels with $95 \%$ confidence level. Descriptive statistics, parametric and non-parametric tests were used appropriately. Paired $t$-test and Kruskal Wallis tests were used. Validation of the criteria has been tested by following two methods.

2.4.1 Validation of the Criteria Is Assessed by Frequent Errors According to Evaluators' Response (Average Comparison between Evaluators' Responses)

Average of the responses given by each evaluator and evaluator category (radiographer and student radiographer) were compared for main and sub categories of the data collection tool to validate the criteria, then the calculated values were used to see if there was any significant difference in observation between each evaluator and evaluator category. Strongly deviated evaluators were excluded. According to that, selected evaluators' final results were given. If the majority gave same answer it suggests criteria were effective and easily understandable.
2.4.2 Validation of the Criteria by the Overall Marks Which Have Given by the Weighted Marks

Above evaluation just used the responses given by the evaluators according to their observation. But differences in errors give different weighting on image quality degradation. Hence the data collection tool was weighted according to the effect of those errors towards the image quality degradation. Maximum mark for each main category was $100 \%$. The data collection tool was given to selected senior radiographers and asked to weight the list. According to the results, marks were finalized (Table 2). Those marks were used for parametric and non-parametric statistical analysis. The image quality was categorized into three groups (excellent quality: over $80 \%$, average quality: between $50 \%-80 \%$, and low quality: below 50\%). Then the outcome of every image was compared among the seven evaluators. Same outcome for majority of the evaluators (more than four) was calculated to comment on the criteria. Fig. 1 and Table 2 show the values given by four qualified radiographers and their average marks. Highly deviated marks were omitted in each sub category and took the average of rest of the marks and justified to get total $100 \%$ for each category.

Table 1 Main categories and sub-categories of the evaluation tool.

\begin{tabular}{ll}
\hline Category & Sub-categories \\
\hline & Name \\
Category 01 (identification) & Sex \\
& Date \\
& Registration number \\
& Anatomical marker \\
\hline Category 02 (artifact) & Foreign body compromise the anatomical area \\
& Motion artifact \\
\hline \multirow{3}{*}{ Category 03 (anatomical coverage) } & Top infra orbit exclusion \\
& Bottom margin is not at the lower border of the mandible \\
& TM joint is not clearly seen \\
\hline & Tongue is not positioned against the palate. \\
& Bite block in not visualizing \\
Lips are not closed \\
Anterior teeth positioning error \\
Incorrect Frankfort plane positioning \\
Head rotated \\
Head tilted \\
Patient is in slumped position (not stand in ski position) \\
Lead apron or thyroid collar positioned too high \\
Motion artifact
\end{tabular}




\section{Results}

\subsection{Comparison of Radiographers and Radiography} Students Image Quality Responses

All seven distributions were tested for normality by Anderson-Darling method. Some of the variables were not normally distributed hence Kruskal Wallis was used as a non-parametric test. The $p$ value of the test was 0.423 which is above the significance level. Therefore there was no significant difference between the responses of the evaluators. Average overall quality marks for radiographers and student radiographers were calculated. Then both distributions were tested for normality in Minitab version 14. Anderson-Darling method was used for normality test. The results showed that the both distributions are normal distributions and both were dependent variables. Therefore paired $t$-test was used to compare the variables. There was no significant difference between the responses of the radiographers and student radiographers as the tested $p$-value (0.000) was above the significance level.

According to the graph majority of the evaluators responded in the same way throughout the sub-categories. Table 2 shows individual evaluator's frequent error according to the average results.

The most frequent error was tongue which is not against the palate that is reported by three evaluators further top infra orbit excluded, lips not closed and bite block is not visualizing said by few evaluators.

\subsection{Individual Responses on Frequent Error-Category Wise}

Table 3, table 4 and table 5 show the individual frequent errors calculated for each category of the data collection tool.

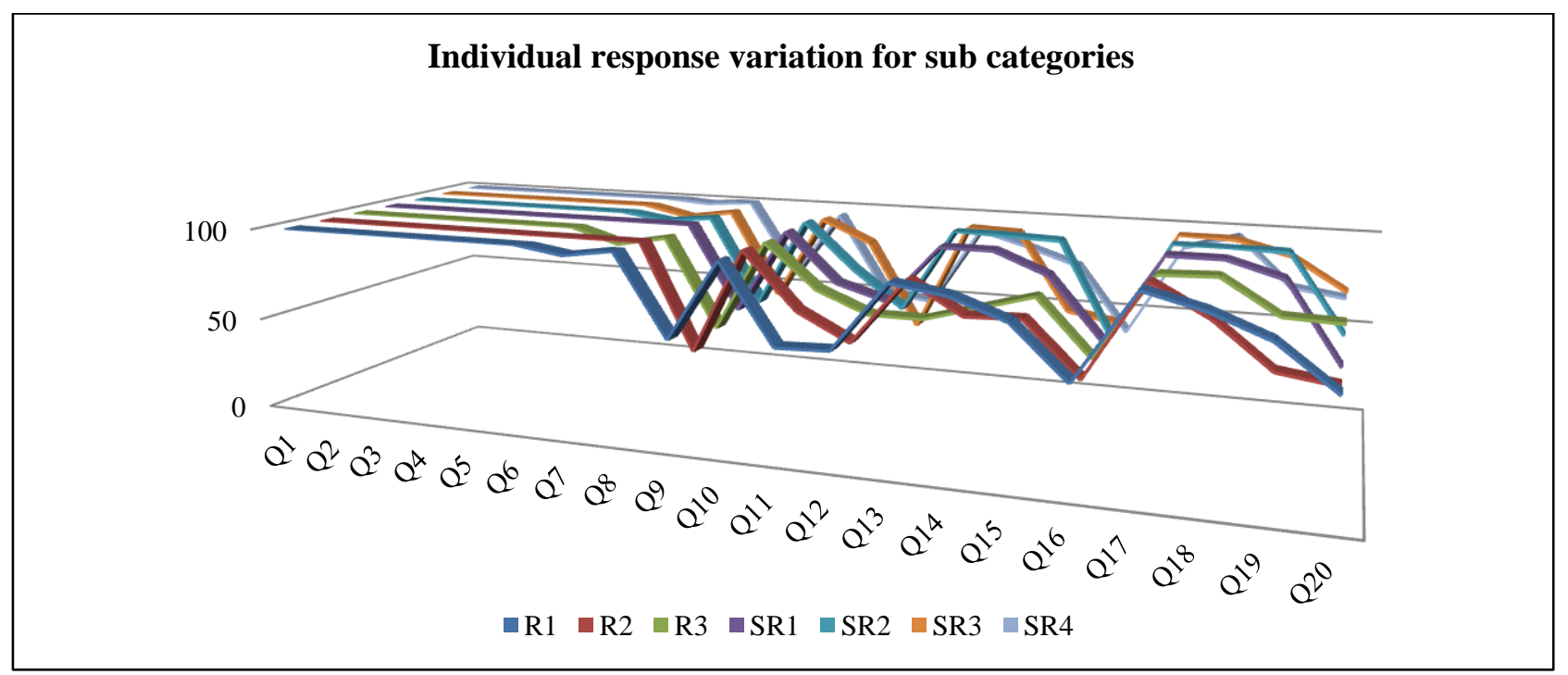

Fig. 1 Individual response variations for sub-categories (R: radiographer, SR: student radiographer).

Table 2 Overall responses on frequent errors (sub-category wise).

\begin{tabular}{lll}
\hline Evaluators & The most frequent error & Second most frequent error \\
\hline R1 & Tongue is not against palate 54\% & Top infra orbit excluded 57\% \\
R2 & Top infra orbit excluded 44\% & Tongue is not against palate $48 \%$ \\
R3 & Top infra orbit excluded 50\% & Tongue is not against palate 51\% \\
SR 1 & Lips not closed 50\% & Tongue is not against palate 53\% \\
SR 2 & Tongue is not against palate 48\% & Top infra orbit excluded \\
SR 3 & Bite block is not visualizing 39\% & Tongue is not against palate 49\% \\
SR 4 & Tongue is not against palate 38\% & Bite block is not visualizing 47\% \\
\hline
\end{tabular}




\section{(OPG) Images in Dental Radiography}

Table 3 Overall responses on frequent errors (sub-category wise)

\begin{tabular}{ll}
\hline Sub category No. & Average response \\
\hline Tongue is not positioned against the palate & $48.7 \%$ \\
Top infra orbit exclusion & $52.7 \%$ \\
Bite block in not visualizing & $55.0 \%$ \\
Lips not closed & $63.4 \%$ \\
TM joint excluded & $69.5 \%$ \\
Anterior teeth positioning error & $81.7 \%$ \\
Incorrect Frankfort plane positioning & $82.2 \%$ \\
Head rotated & $87.1 \%$ \\
Head tilted & $90.2 \%$ \\
Patient is in slumped position (Not stand in ski position) & $94.8 \%$ \\
Foreign body compromise the anatomical area & $96.5 \%$ \\
Lead apron or thyroid collar positioned too high & $96.8 \%$ \\
Bottom margin is not at the lower border of the mandible & $98.1 \%$ \\
Motion artifact & $99.1 \%$ \\
Name & \\
Sex & \\
Age & $100 \%$ \\
Date & \\
Anatomical marker & \\
\hline
\end{tabular}

Table 4 Overall responses on frequent errors of sub-categories according to category.

\begin{tabular}{ll}
\hline Category & Questions \\
\hline Category 01 (identification) & All are same frequently occurring \\
Category 02 (artifact) & Foreign body compromise the anatomical area \\
& Motion artifact \\
Category 03 (anatomical coverage) & TM infra orbit exclusion \\
& Bottom morgin not at the lower border of the mandible \\
& Tongue is not positioned against the palate \\
& Bite block in not visualizing \\
& Lips not closed \\
& Anterior teeth positioning error \\
Category 04 (patient positioning) & Incorrect Frankfort plane positioning \\
& Head rotated \\
Head tilted \\
Patient is in slumped position (not stand in ski position) \\
Lead apron or thyroid collar positioned too high
\end{tabular}

Table 5 Individual responses on frequent error (category wise).

\begin{tabular}{lllllll}
\hline \multicolumn{5}{c}{ Responses of observation (yes values percentages for each main category by each evaluator) } \\
\hline & Category 01 & Category 02 & Category 03 & Category 04 & $\begin{array}{l}\text { Frequent error } \\
\text { category }\end{array}$ & $\begin{array}{l}\text { Second frequent error } \\
\text { category }\end{array}$ \\
\hline R1 & 100 & 98 & 71.3 & 78.7 & Category 03 & Category 04 \\
R2 & 100 & 100 & 71 & 70.8 & Category 04 & Category 03 \\
R3 & 100 & 94.5 & 74.3 & 75.4 & Category 03 & Category 03 \\
SR 1 & 100 & 100 & 75.3 & 81.5 & Category 03 & Category 04 \\
SR 2 & 100 & 97.5 & 75.6 & 84.2 & Category 03 & Category 04 \\
SR3 & 100 & 96 & 77.6 & 81.2 & Category 03 & Category 04 \\
SR4 & 100 & 99 & 68 & 72.5 & Category 03 & Category 04 \\
\hline
\end{tabular}




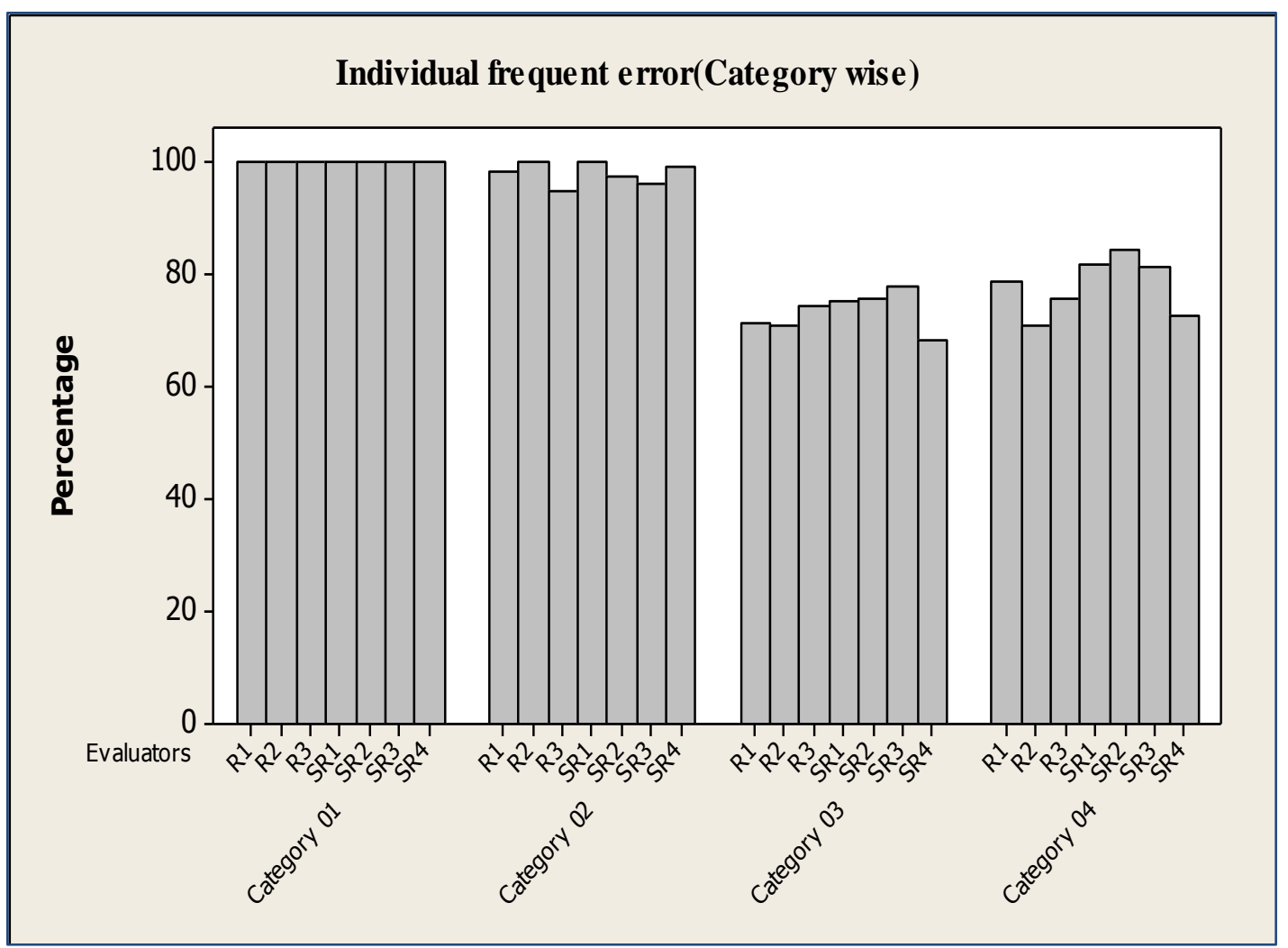

Fig. 2 The comparison between evaluators' responses for each category.

Table 6 Overall responses on frequent error (category wise).

\begin{tabular}{lllllll}
\hline \multicolumn{6}{c}{ Responses of observation (yes values percentages for each main category by each evaluator) } \\
\hline Category 01 & Category 02 & Category 03 & Category 04 & & \\
\hline Average & 100 & 97 & 73.3 & 77.75 & Category 03 & Category 04 \\
\hline
\end{tabular}

Table 7 Overall image quality assessments.

\begin{tabular}{lllll}
\hline \multicolumn{5}{c}{ Same responses (excellent, average or low quality) from the evaluators } \\
\hline Ratio & $7 / 7$ & $6 / 7$ & $5 / 7$ & $4 / 7$ \\
Percentage & 57 & 18 & 10 & 14 \\
\hline
\end{tabular}

Results of the six evaluators showed the most frequent error in Fig. 2 as category 03 (anatomical coverage). Only one person's observation showed that category 04 (patient positioning) as the most frequent error.

Table 6 explains the category 1 (identification) acquired not only the highest mark but also the complete mark. Hence there is no identification error found in the sample. Category 2 (artifact) acquired 97\% value therefore it has a low frequent appearance. But it is the 3rd most frequent error of the sample. Category 3 (anatomical coverage) gained the lowest mark $(73.3 \%)$ according to the evaluation. Therefore it is the highest frequent error category of the data sample. Category4 (patient positioning) acquired $77.7 \%$ and it is the 2 nd most frequent error of the sample.

\subsection{Overall Responses on Image Quality Assessment}

According (Table 7) to the marks on the weighted chart, an overall mark is given for each image for each individual. Excellent quality was above $80 \%$, average quality and poor quality was above $50 \%-80 \%$ and below $50 \%$ respectively. 


\section{Discussion}

The study accessed the validity of the data collection tool by comparing the evaluation responses of each evaluator. In Sri Lanka there was no evidence of already established standard criteria for digital OPG images. A latest study which has been done in Sri Lanka developed an inventory by comparing it to MKC (modified Korean criteria) [2]. Consequently it was vital to develop criteria which were practicable in Sri Lankan technology and other aspects as our country background is not same as other countries. The criteria were developed by referring past literature and edited by well experienced radiographers in Sri Lanka. According to the results overall image quality values have no significant difference between the evaluation of radiographers and student radiographers. Resulted $p$-value was 0.000 which was above 0.05 (significance level). Hence it could interpret in 95\% confidence level. Also there was no significance difference between each evaluator $(p=0.423)$. When considering average values of the responses were given by seven evaluators, it gives the highest frequent error and the least frequent error. The highest mean value gives the least frequent error and the lowest mean value gives the highest frequent error.

Responses of the individual evaluation for each sub-category of the data collection tool showed few sub-categories as the most frequent error for each evaluator. Three of the evaluators' responses showed that the most frequent error as absence of tongue against palate. Responses of radiographer 1 (R1) was $54 \%$, student radiographer 2 (SR2) was $48 \%$ and student radiographer 3 (SR3) was $48 \%$ for the absence of tongue against the palate as the most frequent error. Also response of radiographer 2 (SR2) was 48\%, radiographer 3 was $51 \%$ student radiographer 1 (SR1) was $53 \%$ and student radiographer 3 (SR3) was $49 \%$ for the absence of tongue against the palate as the second most frequent error. According to the results values are not widely deviated from each other.
Similar study on investigation of the most common positioning error showed the absence of tongue against palate as the most frequent error [6].

When considering the average value for all the seven evaluators, the most frequent error was absence of positioning the tongue against the palate which got $41.28 \%$ as the least value. The possible causes of absence of positioning the tongue against the palate as the most frequent error may be due to failure to give proper instruction by the radiographer or failure to follow the instructions. The main disadvantage is that radiographer cannot observe the positioning of tongue hence it may be hard to correct it in many events.

Category of identification showed as the least frequency error of the images and it got total marks. Digital OPG examination required certain patient details to implement the examination. Therefore ignorance of patient details has a low possibility. According to the responses six of the evaluators' observed the most frequent error category as loss of anatomical coverage. The most frequent error category was anatomical coverage but the most frequent error was absent of positioning the tongue against the palate (41.28\%) which was a component in patient positioning. Thus the second and third most frequent errors were components of anatomical coverage. Those achieved $73.3 \%$ and $77.75 \%$ which were not distinct values. Hence it should be influenced to give highest frequent error to anatomical coverage category.

In the last result image quality was represented by a single value. According to those values images were categorized into three categories. Those were excellent quality images, average quality images and low quality images. A similar study has done to investigate image quality of OPG images [7]. The results of the study conveyed the quality outcome of each image was same for each other for $57 \%$ which is more than the half of the images.

\section{Conclusion}

According to the results the selected frequent error 
and overall image quality of radiographer to student radiographers or between evaluators does not change significantly. So the quality evaluation criteria may be an easily understandable and sophisticated one to be used.

\section{References}

[1] Wahab, H. A., Ferguson, D. J., and Kheir, N. A. 2016. "Assessment of Panoral Radiograph Quality in a Dental Treatment Center." Research Gate 6 (2): 85-94.

[2] Nileema, A., Pallegama, R. W., Nuwan, D., and Jayasinghe, R. D. 2016. "Development of a Brief Inventory for Quality Evaluation of Panoramic Radiography." Sri Lanka Dental Journal 45 (2): 55-62.

[3] Choi, B. R., Choi, H, Huh, K. H., Yi, W. J., Heo, M. S., Choi, S. C., Bae, K. H., and Sun, S. L. 2012. "Clinical
Image Quality Evaluation for Panoramic Radiography in Korea Dental Clinics." Imaging Science in Dentistry 42 (3): 183-90.

[4] Vano, E., Guibelaide, E., Morillo, A., Pedrosa, C. S., and Fernandez, J. M. 1995. "Evaluation of European Image Quality Criteria for Chest Examinations." The British Journal of Radiology 68: 1349-55.

[5] Health Physics Society. 2016. Available from http://hps.org/publicinformation/radterms/radfact142.htm 1 (Accessed on 03.01.2017).

[6] Dhillon, M., Raju, S. M., Verma, S., Tomar, D., Mohan, R. S., Lakhanpal, M., and Krishnamoorthy, B. 2012 "Positioning Errors \& Quality Assessment in Panoramic Radiography." Imaging Science in Dentistry 42 (4): 207-12.

[7] Decusara, M., and Miliceseu, V. 2011. "Image Quality Assessment of Orthopantomograms." Romanian Journal of Oral Rehasilitation 3 (4): 54-8. 
(OPG) Images in Dental Radiography

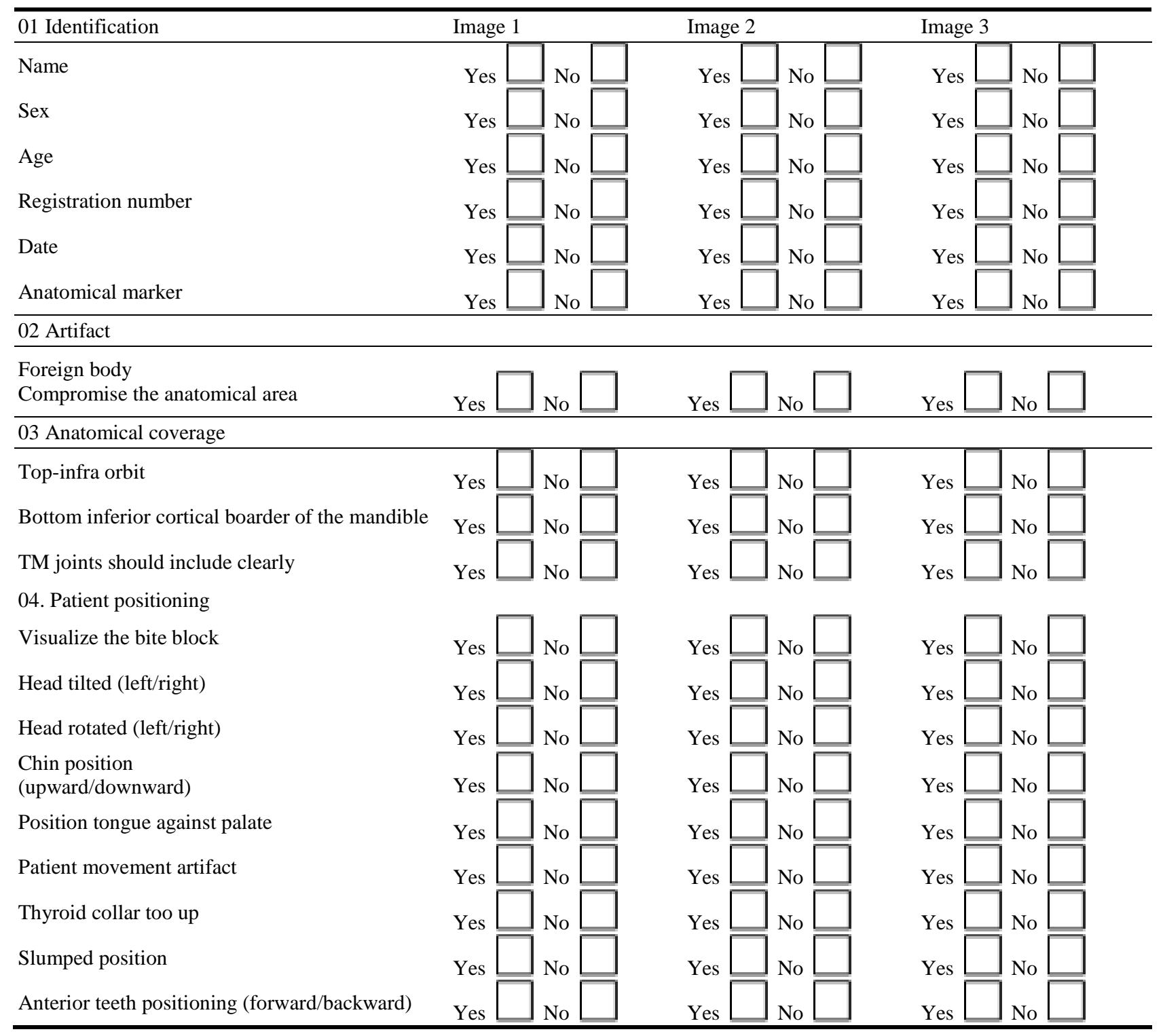

